\begin{tabular}{|c|c|}
\hline Title & $\begin{array}{l}\text { Design of a liquid cell toward three-dimensional imaging of unidirectionally-aligned particles in solution using X-ray } \\
\text { free-electron lasers }\end{array}$ \\
\hline Author(s) & $\begin{array}{l}\text { Suzuki, A kihiro; Kimura, T akashi; Y ang, Y ing; Niida, Y oshiya; Nishioka, A kiko; Tachibana, Tatsuro; Takei, Masashi; } \\
\text { Tono, Kensuke; Y abashi, Makina; Ishikawa, T etsuya; O shima, T ai ro; Bessho, Y oshitaka; Joti, Y asumasa; Nishino, } \\
\text { Y oshinori }\end{array}$ \\
\hline Citation & $\begin{array}{l}\text { Physical chemistry chemical physics, 22(5), 2622-2628 } \\
\text { https://doi.org/10.1039/c9cp03658j }\end{array}$ \\
\hline Issue Date & $2020-02-07$ \\
\hline Doc URL & http:/hdl.handle.net/2115/80381 \\
\hline Type & article (author version) \\
\hline File Information & tiltMLEA_final.pdf \\
\hline
\end{tabular}

Instructions for use 
Received 28th June 2019, Accepted 14th August 2019 DOI: $10.1039 /$ c9cp03658j

\title{
Design of liquid cell toward three-dimensional imaging of unidirectionally-aligned particles in solution using $\mathrm{X}$-ray free- electron lasers
}

\author{
Akihiro Suzuki, ${ }^{a}$ Takashi Kimura, ta Ying Yang, ${ }^{a}$ Yoshiya Niida, ${ }^{a}$ Akiko Nishioka, ${ }^{a}$ Tatsuro Tachibana, ${ }^{a}$ \\ Masashi Takei, ${ }^{a}$ Kensuke Tono, ${ }^{\mathrm{b}}$ Makina Yabashi, ${ }^{\mathrm{c}}$ Tetsuya Ishikawa, ${ }^{\mathrm{c}}$ Tairo Oshima, ${ }^{\mathrm{d}}$ Yoshitaka \\ Bessho, ${ }^{\text {ce }}$ Yasumasa Joti ${ }^{\mathrm{b}}$ and Yoshinori Nishino*a
}

\begin{abstract}
X-ray free-electron lasers (XFELs) opened up a possibility for molecular-scale single particle imaging (SPI) without the need for crystallization. In SPI experiments, the orientation of each particle has to be determined from the measured diffraction pattern. Preparing unidirectionally-aligned particles can facilitate the determination of sample orientation. Here, we show design principles of a liquid cell for three-dimensional imaging of unidirectionally-aligned particle in solution with XFELs. The liquid cell was designed so that neither incident X-rays nor diffracted X-rays are blocked by the substrate of the liquid cell even at high tilt angles. As a feasibility evaluation, we performed coherent diffraction measurement using the cells with a 1$\mu \mathrm{m}$ focused XFEL beam. We successfully measured coherent diffraction patterns of a nano-fabricated metal pattern at $70^{\circ}$ tilt angle and obtained the reconstructed image by applying iterative phase retrieval. The liquid cell will be usefully applied to molecular-scale SPI by using more tightly focused XFELs. In particular, imaging of membrane proteins embedded in lipid membranes is expected to have an enormous impact on life science and medicine.
\end{abstract}

\section{Introduction}

Femtosecond-pulsed X-ray free-electron lasers (XFELs) enable measurement free from radiation damage with the diffractionbefore-destruction strategy. ${ }^{1,2}$ In addition, the almost-perfect spatial coherence of XFELs is favorable for coherent diffractive imaging (CDI) experiments. ${ }^{3} \mathrm{CDI}$ allows us to image noncrystalline sample, thus is expected to provide a breakthrough in single particle imaging (SPI) of biomolecules ${ }^{4}$ that are difficult to crystallize, such as membrane proteins. CDI measurement with XFELs can be performed at room temperature by overcoming the radiation damage problem, and thus has advantages also in studying structural dynamics of biological targets.

Three-dimensional SPI realizes by collecting coherent diffraction patterns of particles with a reproducible structure at different incident angles. In SPI experiments, it is necessary to determine the particle orientation from the measured coherent

\footnotetext{
a. Address here. Research Institute for Electronic Science, Hokkaido University, Kita 21 Nishi 10, Kita-ku, Sapporo 001-0021, Japan.

E-mail: yoshinori.nishino@es.hokudai.ac.jp

b. Japan Synchrotron Radiation Research Institute/SPring-8, 1-1-1 Kouto, Sayo-cho,

Sayo-gun, Hyogo 679-5198, Japan

c. RIKEN SPring-8 Center, 1-1-1 Kouto, Sayo-cho, Sayo-gun, Hyogo 679-5148, Japan.

d. Institute of Environmental Microbiology, Kyowa-kako Co. Ltd., 2-15-5 Tadao,

Machida, Tokyo 194-0035, Japan

e. Institute of Biological Chemistry, Academia Sinica, 128, Academia Road Sec. 2,

Nankang, Taipei 115, Taiwan

+ Present address: Department of Precision Engineering, Graduate School of Engineering, The University of Tokyo, Hongo 7-3-1, Bunkyo-ku, Tokyo 113-8656, Japan
}

diffraction patterns. ${ }^{5,6}$ In aerosol injectors widely used in SPI experiments, 7,8 the particle orientation is usually random. In fixed target experiments, it is also possible to prepare unidirectionally-aligned particles.

The determination of the sample particle orientation from the measured coherent diffraction pattern will become simpler for unidirectionally-aligned particles as compared to randomly oriented particles. It is also expected that a smaller number of diffraction patterns will be required for three-dimensional imaging when measuring unidirectionally-aligned particles.

In the fluctuation scattering method, single-particle diffraction data can be calculated by taking angular correlations of many coherent diffraction patterns, and three-dimensional images can be reconstructed subsequently. ${ }^{9,10}$ Fluctuation scattering can also be performed even when illuminating more than one particle in coherent diffraction measurement assuming that inter-particle interference can be neglected. ${ }^{11,12}$ Illumination of more than one particle helps to enhance the diffraction signal and will lead to a higher spatial resolution in particle imaging. Elser pointed out that $a b$ initio threedimensional imaging in fluctuation scattering generally requires unidirectionally-aligned particles, because there are information deficits for randomly oriented particles. ${ }^{13}$

When measuring unidirectionally-aligned particles, one needs to rotate the sample using an automated stage to measure coherent diffraction patterns at different tilt angles. In SPI measurement, neither incident nor diffracted X-rays should be blocked by the sample holder. This article describes the design principles of a liquid cell specialized for coherent 
diffraction measurement at high tilt angles toward threedimensional imaging of unidirectionally-aligned particles.

\section{Pulsed coherent X-ray solution scattering (PCXSS)}

Liquid cells have been employed in fixed-target CDI using XFELs and the measurement technique is referred to as pulsed coherent X-ray solution scattering (PCXSS). ${ }^{14,15}$ The authors' group has successfully applied PCXSS to snapshot imaging of live cells and open the application of XFEL imaging to bacterial cell biology. ${ }^{15}$ PCXSS is also useful in imaging inorganic particles that can maintain their structure in solution only. ${ }^{16,17}$

In PCXSS measurement using SACLA, micrometer-focused XFEL beam has been used to image sub-micrometer-sized targets. MAXIC (multiple application X-ray imaging chamber) ${ }^{18}$ and the successor MAXIC-II have been used as sample chambers. For molecular-scale SPI at higher spatial resolution, it is required to increase XFEL fluence to enhance the diffraction signal at higher scattering angles. The SACLA facility has recently developed MAXIC-S for SPI utilizing 100-nm focused XFEL in collaboration with the authors' group. ${ }^{19}$ MAXIC-S is open for public users since 2018B SACLA beamtime.

In PCXSS measurement, MLEAs (micro-liquid enclosure arrays) have been used to hold solution samples. ${ }^{20}$ In MLEAs, the solution sample is sandwiched in between two freestanding thin membranes, each of which is supported by a silicon substrate. The membranes act as windows to illuminate the sample and to pass the diffracted X-rays. Since each microliquid enclosure is destroyed with an intense single XFEL pulse, many enclosures are integrated into a single MLEA and are shot with XFEL pulses one by one. MLEAs are fabricated by photolithography in-house at Hokkaido University.

The simplest way to enclose a solution sample in an MLEA is to disperse particles in solution. In this case, the particle orientations are random as in the case of aerosol injectors. The authors are also considering to enclose unidirectionally-aligned particles in MLEAs by employing various soft lithography techniques. ${ }^{21}$ Those techniques are widely used in biology and biochemistry and are applied for, e.g., localized surface plasmonic resonance (LSPR) sensors ${ }^{22}$ and high-speed atomic force microscopy. ${ }^{23}$ For example, a self-assembled monolayer $(\mathrm{SAMs})^{24}$ on a membrane window can be used to attach the sample via biological tags, such as the histidine tag. It is also possible to fabricate lipid bilayer chambers in the MLEA and embed membrane proteins in the lipid bilayer while maintaining their activities. ${ }^{25}$

\section{Micro-liquid enclosure arrays (MLEAs)}

The MLEAs for high tilt angles are designed based on the ones that have been used for PCXSS measurement. Therefore, we first briefly explain the design and fabrication process of existing MLEAs. MLEAs are made from silicon substrates coated with thin silicon nitride ( $\mathrm{SiN}$ ) on both sides. An array of free-standing SiN membrane windows is fabricated by photolithography using $\mathrm{KOH}$ etching of silicon. MLEAs are assembled by sticking two silicon substrates with free-standing SiN membrane windows after precise alignment.

The independence of each micro-liquid enclosure in an MLEA is secured by the grid partition fabricated on one of the silicon substrates by photolithography. The thickness of the liquid layer can be determined by the height of the grid partition. The widths of the window aperture are currently $20 \mu \mathrm{m}$ in both the directions, which is enough larger than the 1- $\mu \mathrm{m}$ beam size of focused XFELs. Tapered silicon frames of the X-ray illuminating window act as slit blades and have been proven to be effective in reducing parasitic scattering from upstream optics. ${ }^{15}$

$\mathrm{KOH}$ etching is highly anisotropic: the etch rate in the $<111>$ direction is slower than in the $<100>$ direction by two or three orders of magnitude depending on the condition. Therefore, when using silicon substrates with the (100) surface, a tapered structure with a slope of $54.7^{\circ}$ is formed in $\mathrm{KOH}$ etching. Due to the tapered structure, the etched area is much larger at the backside of the substrate, and thinner silicon substrates are required to shorten the interval between windows for a higher degree of integration. Conversely, thinner silicon substrates increase the risk of breakage them in handling. The thickness of silicon we are currently using is $150 \mu \mathrm{m}$. The SiN membrane has a thickness of $200 \mathrm{~nm}$, which is relatively thick in order not to break it during the fabricating process of the grid partition after $\mathrm{KOH}$ etching and the subsequent cleaning process.

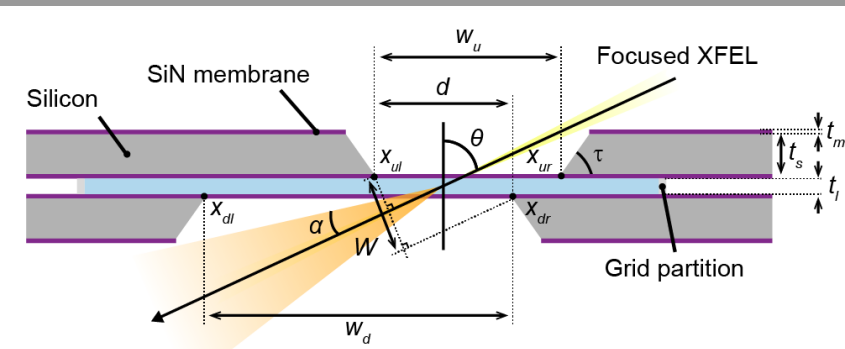

Fig. 1 Schematic cross-section of an MLEA for high tilt angles. $\alpha$ is the tilt angle of the XFEL beam, $\theta$ is the maximum diffraction angle, and $\tau$ is the taper angle of the silicon substrate. $t_{l}, t_{s}$ and $t_{m}$ are the thickness of the liquid layer, the silicon substrate and the SiN membrane, respectively. The widths of the upstream and downstream windows are given by $w_{u}$ and $w_{d}$, respectively. $d$ is the window overlap. $W$ is the effective slit width as seen from the XFEL beam.

\section{MLEAs for high tilt angles}

\section{General design}

In designing MLEAs for high tilt angles, the first thing we should consider is to prevent the incident and diffracted X-rays from hitting the substrate of MLEAs. Fig. 1 shows the cross-sectional structure of an MLEA for high tilt angles we designed. The upstream and downstream substrates have different window widths, and the centers are shifted with some overlap. In Fig. 1, $\alpha, \theta$ and $\tau$ are the tilt angle of the XFEL beam, the maximum diffraction angle considered and the taper angle of the silicon substrate, respectively. $t_{l}, t_{s}$ and $t_{m}$ are the thicknesses of the liquid layer, the silicon substrate and the SiN membrane, 
respectively. For the given experimental condition set by the above six variables, we determine the window widths and the window overlap.

We take the horizontal directions of Fig. 1 as the $x$-axis, and the direction to the right is positive $x$. The $x$-coordinates of the left and right edges of the upstream windows are $x_{u l}$ and $x_{u r}$, respectively. Similarly, the coordinates of the left and right edges of the downstream windows are $x_{d l}$ and $x_{d r}$. respectively. Then, the widths of the upstream and downstream windows are given by $w_{u}=x_{u r}-x_{u l}$ and $w_{d}=x_{d r}-x_{d l}$, respectively. The window overlap is defined by $d=x_{d r}-x_{u l}$.

We first consider the window overlap $d$. We assume the XFEL beam passes through the $x$-coordinate $\left(x_{u l}+x_{d r}\right) / 2$ at the middle of the liquid layer and set the coordinate to the origin: $\left(x_{u l}+x_{d r}\right) / 2=0$. Then we have $x_{d r}=-x_{u l}=d / 2$. The effective slit width $W$ as seen from the XFEL beam is given by

$$
W=\sqrt{d^{2}+\left(t_{l}+2 t_{m}\right)^{2}} \cos \left(\alpha-\operatorname{atan}\left(\frac{t_{l}+2 t_{m}}{d}\right)\right) .
$$

For $d \gg\left(t_{l}+2 t_{m}\right)$, it is simply given by $W=d \cos \alpha$. For a given tilt angle $\alpha$, the window overlap $d$ can be determined from the desired effective slit width $W$ using Eq. (1).

Next, we consider the window widths $w_{u}$ and $w_{d}$. The condition to prevent the incident XFEL beam from hitting the upstream substrate is that, at the top surface of the upstream substrate, the $x$-coordinate $\left(x_{u r}+t_{s} \cot \tau\right)$ of the right tapered frame of the window is larger (more to the right) than the $x$ coordinate $\left(t_{s}+2 t_{m}+t_{l} / 2\right) \tan \alpha$ of the incident XFEL beam. Thus, we have

$$
x_{u r}=w_{u}-\frac{d}{2}>\left(t_{s}+2 t_{m}+\frac{t_{l}}{2}\right) \tan \alpha-t_{s} \cot \tau .
$$

Similarly, the condition to prevent the diffracted XFEL beam from hitting the downstream substrate is that, at the bottom surface of the downstream substrate, the $x$-coordinate $\left(x_{d l}-t_{s} \cot \tau\right)$ of the left tapered frame of the window is smaller (more to the left) than the $x$-coordinate $-\left(t_{s}+2 t_{m}+t_{l} / 2\right) \tan (\alpha+\theta)$ of the left edge of the diffracted XFEL beam. Thus, we have

$$
x_{d l}=-w_{d}+\frac{d}{2}<-\left(t_{s}+2 t_{m}+\frac{t_{l}}{2}\right) \tan (\alpha+\theta)+t_{s} \cot \tau .
$$

The thicknesses of the liquid layer $t_{l}$ and the membrane $t_{m}$ are usually much thinner than the thickness of the silicon $t_{s}$, thus $\left(t_{s}+2 t_{m}+t_{l} / 2\right)$ in Eqs. (2) and (3) can be approximated by $t_{s}$.

\section{Specific design for MAXIC-S measurement}

Tomography measurement at high tilt angles is generally hindered by the sample holder, which causes missing data. The problem is known as the missing wedge and can causes artefacts in tomographic reconstruction. ${ }^{26}$ The missing wedges should be minimized for faithful image reconstruction, although the iterative phase retrieval algorithm help recover the missing diffraction data in three-dimensional CDI.
Measurement at higher tilt angles requires the increase of the window widths, especially the downstream window width. Wider windows result in a lower degree of integration, i.e. a smaller number of micro-liquid enclosures in a single MLEA. Larger tilt angle also makes the $\mathrm{X}$-ray footprint on the liquid cell plane larger, which causes a decrease in the incident X-ray fluence. Furthermore, to keep the number of particles in the XFEL interaction volume constant (e.g., to maximize singleparticle hits), a larger X-ray footprint requires a more diluted sample solution. In this study, we assume $70^{\circ}$ to be the maximum tilt angle in considering the above-mentioned technical limitations and previous studies of successful threedimensional CDI. ${ }^{27}$

Now we consider the maximum diffraction angle for the experimental setup using MAXIC-S. In the setup, diffraction patterns are recorded by the MPCCD (multi-port chargecoupled device) ${ }^{28}$ octal sensor detector located at $320 \mathrm{~mm}$ downstream of the sample. Each of 8 sensors of the MPCCD octal sensor detector has $1024 \times 512$ pixels, and 8 sensors are tiled to make a total of $2048 \times 2048$ pixels with a single pixel size of $50 \mu \mathrm{m}$. The MPCCD octal sensor detector has an open aperture at the middle with a maximum aperture size of $9.9 \mathrm{~mm}$ and the diffracted $\mathrm{X}$-ray passed through the aperture can be recorded with the MPCCD dual sensor located $1600 \mathrm{~mm}$ downstream of the sample. For the maximum aperture size of $9.9 \mathrm{~mm}$, the side length of the MPCCD octal sensor detector is $112.3 \mathrm{~mm}$. This corresponds to the diffraction angle $\theta=10^{\circ}$ at the edge of the MPCCD octal sensor detector. We take this value as the maximum diffraction angle in designing MLEAs for high tilt angles.

The achievable spatial resolution $\delta$ is determined by the wavelength $\lambda$ of the XFEL beam and the maximum diffraction angle $\theta$ as $\delta=\lambda /(2 \sin (\theta / 2))$. MAXIC-S is designed for an XFEL wavelength of $3.1 \AA$ or a photon energy of $4 \mathrm{keV}$. Then the achievable resolution using MAXIC-S is given by $1.8 \mathrm{~nm}$ at the edge of the MPCCD octal sensor detector.

From the above considerations, we designed MLEAs for high tilt angles as discussed below. We set the thicknesses of the liquid layer, silicon and $\mathrm{SiN}$ membrane to $t_{l}=200 \mathrm{~nm}$, $t_{s}=150 \mu \mathrm{m}$ and $t_{m}=200 \mathrm{~nm}$, respectively. The tilt angle of XFEL, the maximum diffraction angle considered and the taper angle of the substrate are set to $\alpha=70^{\circ}, \theta=10^{\circ}$ and $\tau=54.7^{\circ}$, respectively.

We set $W$ to around $20 \mu \mathrm{m}$ as practiced in our normal incidence measurement using MAXIC and MAXIC-II. By substituting the parameter values in Eq. (1), we have $d=57 \mu \mathrm{m}$ for $W=20 \mu \mathrm{m}$. We set the window overlap to $d=60 \mu \mathrm{m}$ and the short side width of both windows to $20 \mu \mathrm{m}$, which makes the effective slit size around $20 \mu \mathrm{m}$ in both directions.

By substituting the parameter values in Eqs. (2) and (3), we have $w_{u}>337 \mu \mathrm{m}$ and $w_{d}>777 \mu \mathrm{m}$, respectively. In the actual design, the long side widths of upstream and downstream windows are set to $w_{u}=350 \mu \mathrm{m}$ and $w_{d}=880 \mu \mathrm{m}$, respectively. The upstream and downstream substrates have a square shape and the side lengths are $12 \mathrm{~mm}$ and $14 \mathrm{~mm}$, respectively. The downstream substrate is larger because of the margin for gluing with ultraviolet resin after tight sticking of the two substrates. 
Fig. 2(a) show a picture of MLEAs for high tilt angles we fabricated and Fig. 2(b) an enlarged view of a single window. The fabricated MLEA has 24 windows in one-dimensional array at an interval of $350 \mu \mathrm{m}$. The degree of integration of MLEAs can be easily improved by making two-dimensional arrays of windows. The fabrication and assembly processes of MLEAs are the same as for the standard MLEAs and the details are described in a separate paper. ${ }^{20}$
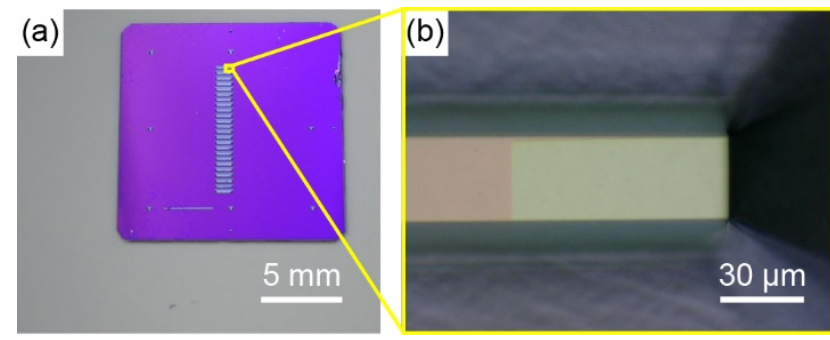

Fig. 2. The digital microscope images of an MLEA for high tilt angles. (a) Whole picture and (b) enlarged view of a single window.

\section{Evaluation of MLEAs for high tilt angles}

\section{Fabrication of metal test pattern sample}

To evaluate MLEAs for high tilt angles, we performed CDI measurement using a test sample composed of a twodimensional array of gold cylinders on the SiN windows. No liquid was enclosed in the cell in the demonstration measurement. Fig. 3 show the design of the test sample. Each cylinder is $200 \mathrm{~nm}$ in diameter and $50 \mathrm{~nm}$ in thickness. As for the interval between cylinders, we designed so that a single cylinder is illuminated by the focused XFEL beam with a focal spot size of $\sim 1.5 \mu \mathrm{m}$ (full-width at half maximum). For higher tilt angles, $\mathrm{X}$-ray footprint on the SiN window becomes longer by a factor of $1 / \cos \alpha$ in the direction perpendicular to the rotation axis, and it becomes $4.4 \mu \mathrm{m}$ long for $70^{\circ}$ tilt angle. From the above consideration, for $70^{\circ}$ tilt angle, we set the interval between the cylinders to $5 \mu \mathrm{m}$ and $15 \mu \mathrm{m}$ in the direction parallel and perpendicular to the rotation axis, respectively.

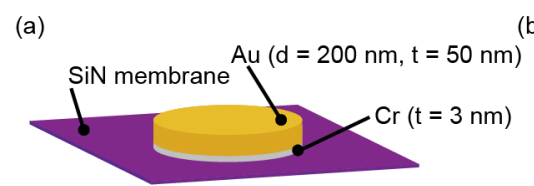

(b) Gold cylinders

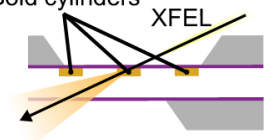

Fig. 3. The design of a cylindrical object used as a test sample. (a) The diameter and thickness of gold are $200 \mathrm{~nm}$ and $50 \mathrm{~nm}$, respectively. Chromium is used as an adhesion promoter. (b) A gold cylinder is fabricated on the upstream SiN window.

We fabricated the test pattern sample by electron lithography using ELS-F125 (ELIONIX Inc.) at $125 \mathrm{kV}$. As a highresolution positive resist, we used ZEP-520A (ZEON Corp.). In order to render electrical conductivity to the surface of the resist, ESPACER $300 Z$ (SHOWA DENKO) was spin coated. Although ultrasonic agitation is often used in the lift-off process, we have to avoid it to prevent the thin SiN window from breaking. Instead, we stationarily immersed the substrates into a remover solution $Z D M A C$ at $130^{\circ} \mathrm{C}$ for 10 minutes followed by a rinse with acetone, methanol and distilled water. Fig. 4(a) shows a top view of the sample imaged by confocal laser microscopy (CLM). This image shows that nanometer-scale objects were formed at the designed interval. Fig. 4(b) shows a scanning electron microscopy (SEM) image of a single cylinder thus fabricated. Because backscattered electrons from the substrate also exposed the resist, the diameter of the fabricated cylinder is larger than the designed one by about $30 \mathrm{~nm}$. The SEM image in Fig. 4(b) is vertically elongated presumably due to the positional drift of electron beam in SEM observation caused by the charging effect, whereas the actual structure should be circular.

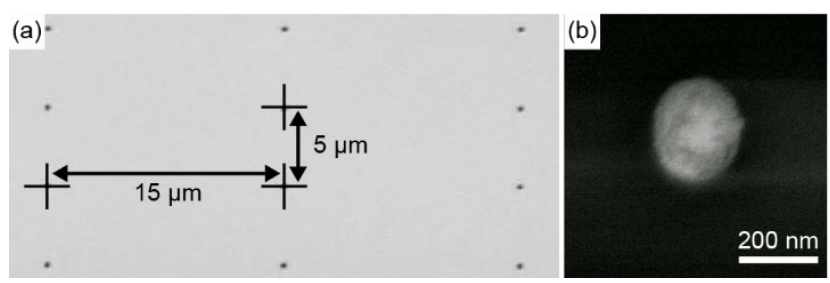

Fig. 4. Top views of the test pattern sample used for $C D I$ measurement at $70^{\circ}$ tilt angle. (a) a confocal laser microscopy (CLM) image of an array of Au cylinders and (b) a scanning electron microscopy (SEM) image of a single Au cylinder.

\section{CDI measurement with MAXIC}

We brought the test sample to BL3 of SACLA and performed CDI measurement using MAXIC. MAXIC was slowly evacuated to prevent the thin SiN windows of MLEAs from breaking. The XFEL beam from SACLA with a photon energy of $4 \mathrm{keV}$ was guided to $\mathrm{EH} 4 \mathrm{c}$ after removing higher harmonics with a total reflection double mirror system at the optics hutch. In EH4c, the XFEL beam was focused to a spot size of $\sim 1.5 \mu \mathrm{m}$ with a KirkpatrickBaez mirror system ${ }^{29}$ and illuminated the sample at the focal position in MAXIC. ${ }^{18}$ Diffraction patters were recorded with an MPCCD octal sensor detector located $1.525 \mathrm{~m}$ downstream of the sample. Although MAXIC-S is equipped with a sample rotation stage, MAXIC used for the test measurement isn't. We therefore made a special MLEA holder with fixed tilt angles as the three-dimensional drawing shown in Fig. 5(a). Fig. 5(b) is a picture of the MLEA holder in MAXIC.

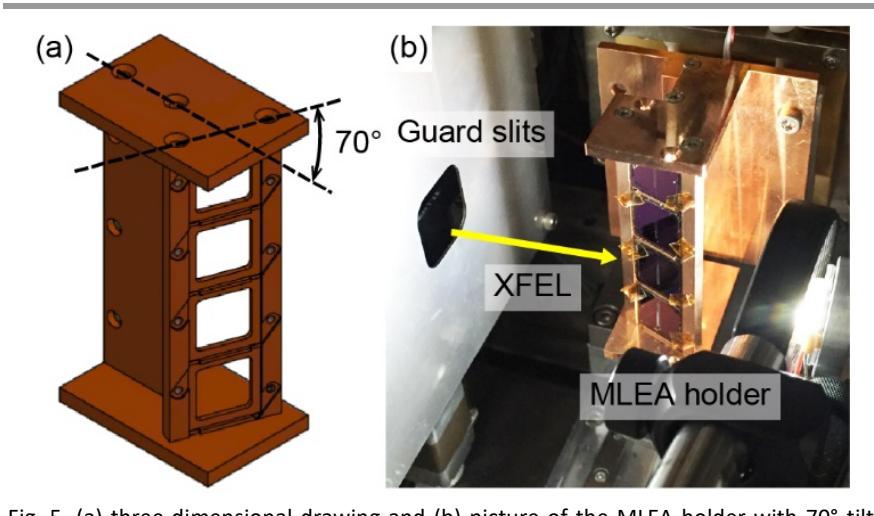

Fig. 5. (a) three-dimensional drawing and (b) picture of the MLEA holder with $70^{\circ}$ tilt angle.

In aligning the window aperture to the XFEL beam, we have to take a different method from that used for normal incidence 
measurement. In normal incidence measurement, we exposed the silicon frame of an MLEA with a single XFEL pulse and observed the damaged spot with the zoom lens camera to determine the XFEL beam position on the camera monitor screen. Here, we used epi-illumination and observed the reflected light from the MLEA. In tilt angle measurement, the reflected light did not enter the camera and therefore we could not observe MLEAs with the zoom lens camera. Instead, we illuminated the windows at the corners of the MLEA with an attenuated XFEL beam and monitored the transmitted X-ray intensity with a PIN photodiode to determine the center coordinates. The positions of the other windows were determined by interpolation.

\section{Image reconstruction}

Figs. 6. (a) and (b) show the diffraction patterns of the test sample at tilt angles of $0^{\circ}$ and $70^{\circ}$, respectively. (c) and (d) are sample images reconstructed from (a) and (b), respectively. (e) shows the ideal projection image of the cylinder pattern at a tilt angle of $70^{\circ}$. We successfully recorded coherent diffraction patterns even at a tilt angle of $70^{\circ}$ without any blocking. The coherent diffraction intensities extend to the edges and the corners of the MPCCD octal sensor detector, where the spatial resolution corresponds to $6 \mathrm{~nm}$ at the corners. All diffraction patterns measured were from single isolated cylinders as we designed, enabling image reconstruction. The reconstructed sample image at $70^{\circ}$ in Fig. $6(\mathrm{~d})$ has a size consistent with the ideal projection shown in Fig. 6(e). The non-uniformity of the reconstructed image intensity, which can be also seen in the SEM image of Fig. 4(b), is expected to be induced by the inaccuracy in electron-beam lithography.

Image reconstruction was carried out on the HPC system at SACLA. ${ }^{30}$ To improve the signal-to-noise ratio of the diffraction data, the centrosymmetrized diffraction pattern was first $4 \times 4$ binned, which corresponds to the one-dimensional oversampling ratio $\sim 8$, and cropped into $521 \times 521$ pixels. The centrosymmetrization of the diffraction pattern can be justified in considering that the sample is composed mostly of gold and the maximum phase shift is 0.74 radian even at $70^{\circ}$ tilt angle for $4 \mathrm{keV} \mathrm{X}$-rays. The reconstruction consisted of 10,000 iterations with the hybrid-input-output algorithm ${ }^{31}$ coupled with shrinkwrap algorithm ${ }^{32}$ and oversampling smoothness algorithm. ${ }^{33}$ The initial support was obtained from the threshold applied to the autocorrelation of the object. ${ }^{32}$ The feedback parameter of shrink-wrap algorithm was set to 0.89 and the support was updated every 20 iterations with a threshold of 0.05 . In addition, a Gaussian filter function was multiplied to the density outside the support and its width was changed linearly every 1000 iterations. Each iteration was repeated 500 times with independent and random starting density maps, and the 54 (tilt angle of $0^{\circ}$ ) or $178\left(70^{\circ}\right)$ good quality images with high similarity (correlation coefficient $>0.96$ ) were selected and averaged to yield the final reconstruction. The spatial resolution of the reconstructed images shown in Figs. 6 (c) and (d) are $20 \mathrm{~nm}$ and $21 \mathrm{~nm}$, respectively, as estimated using the phase retrieval transfer function (PRTF) ${ }^{34}$ with a threshold value of $1 / e$ (Fig. 6 (f)).
The above demonstration experiment is for strongly scattering gold patterns. However, there remain technical challenges to be tackled for molecular-scale SPI of biological targets mainly because of the weakness of the scattering signal. Increase in the pulse energy of XFEL sources always help enhance the scattering signal. As a measure for weak scattering signal, it is also effective to illuminate multiple sample particles and apply the fluctuation scattering technique..$^{9^{-13}}$ Although SPI using liquid cells gives an additional challenge of, e.g., reduced image contrast due to the surrounding solvent, it has significant advantages for detailed analysis of biomolecular dynamics owing to the capability of measurement under controlled environmental conditions. Furthermore, the incorporation of lipid bilayer chambers ${ }^{25}$ into the liquid cells will allow the analysis of active membrane proteins under physiological conditions. Efforts toward molecular-scale SPI are underway at XFEL facilities over the world. ${ }^{35}$

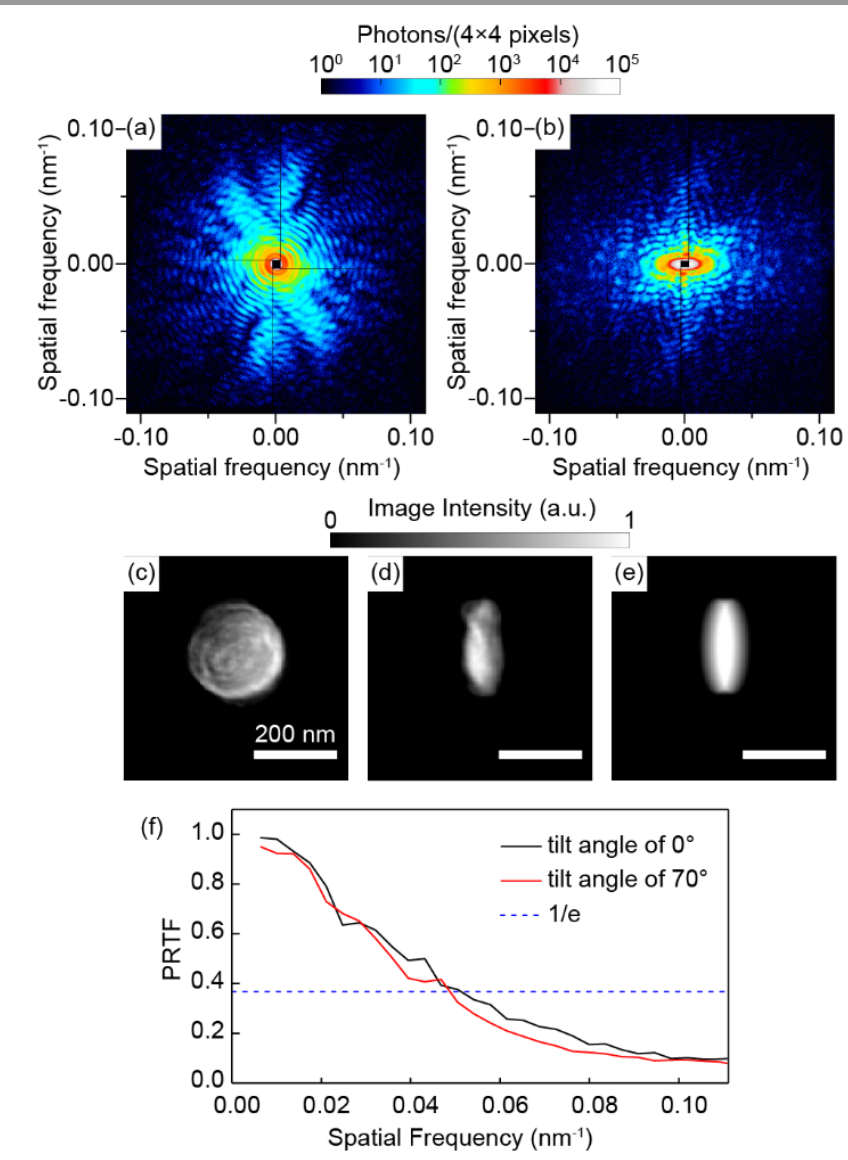

Fig. 6. (a) and (b) diffraction patterns of the test sample at tilt angles of $0^{\circ}$ and $70^{\circ}$ respectively. (c) and (d) are sample image reconstructed from (a) and (b), respectively. (e) the ideal projection image of the cylindrical object with $53 \mathrm{~nm}$ thickness at a tilt angle of $70^{\circ}$. (f) Phase-retrieval transfer function (PRTF) of the reconstructed images shown in (c) and (d). The spatial resolution of the both images is $\sim 20 \mathrm{~nm}$ as estimated from the spatial frequency, where the PRTF drops to $1 / e$

\section{Conclusion}

We showed the design principles of MLEAs for SPI of unidirectionally aligned particles at high tilt angles. MLEAs for high tilt angles were designed so that neither incident and 
diffracted X-ray are blocked. We performed CDI measurement to evaluate them using the 1- $\mu \mathrm{m}$ focused XFEL beam at SACLA. Using a test sample composed of a two-dimensional array of nanofabricated gold cylinders, we demonstrated the successful coherent diffraction measurement and subsequent image reconstruction at $70^{\circ}$ tilt angle. The equations shown in this article are general and can be used also in optimizing the design of MLEAs for molecular level imaging of unidirectionally aligned particles using the $100-\mathrm{nm}$ focused XFEL beam offered by MAXIC-S. As an example of future targets of unidirectionally aligned particles, membrane proteins embedded in lipid bilayer chambers have significance in life science and medicine.

\section{Conflicts of interest}

There are no conflicts of interest to declare.

\section{Acknowledgements}

The XFEL experiments were performed at the BL3 of SACLA with the approval of the Japan Synchrotron Radiation Research Institute (JASRI) (Proposal Nos. 2017B8061, 2017A8006, 2016B8065, 2016A8049 and 2015B8050). The phase retrieval analysis was carried out using the Mini-K supercomputer and HPC system at the SACLA facility (Proposal Nos. 2017B8061, 2017A8006, 2016B8065 and 2016A8049). We thank the operation and engineering staff of SACLA for helping perform the PCXSS experiment; the Nanotechnology Platform Program of MEXT for helping MLEA fabrication; M. Kusuzaki for fabricating jigs to seal MLEAs.

This work was supported by JSPS KAKENHI 15H05737, 16H05989, 16K01942, 16K05527 and 17H04819; the X-ray Free Electron Laser Priority Strategy Program of MEXT; and the Research Program of "Five-star Alliance" and the Cooperative Research Program of "NJRC Mater. \& Dev."

\section{Notes and references}

1 R. Neutze, R. Wouts, D. van der Spoel, E. Weckert and J. Hajdu, Nature, 2000, 406, 752-757.

2 H. N. Chapman, A. Barty, M. J. Bogan, S. Boutet, M. Frank, S. P. Hau-Riege, S. Marchesini, B. W. Woods, S. Bajt, W. H. Benner, R. A. London, E. Plönjes, M. Kuhlmann, R. Treusch, S. Düsterer, T. Tschentscher, J. R. Schneider, E. Spiller, T. Möller, C. Bostedt, M. Hoener, D. A. Shapiro, K. O. Hodgson, D. van der Spoel, F. Burmeister, M. Bergh, C. Caleman, G. Huldt, M. M. Seibert, F. R. N. C. Maia, R. W. Lee, A. Szöke, N. Timneanu and J. Hajdu, Nat. Phys., 2006, 2, 839-43.

3 J. Miao, P. Charalambous, J. Kirz and D. Sayre, Nature, 1999, 400, 342-344.

4 A. Aquila, A. Barty, C. Bostedt, S. Boutet, G. Carini, D. dePonte, P. Drell, S. Doniach, K. H. Downing, T. Earnest, H. Elmlund, V. Elser, M. Gühr, J. Hajdu, J. Hastings, S. P. Hau-Riege, Z. Huang, E. E. Lattman, F. R. N. C. Maia, S. Marchesini, A. Ourmazd, C. Pellegrini, R. Santra, I. Schlichting, C. Schroer, J. C. H. Spence, I. A. Vartanyants, S. Wakatsuki, W. I. Weis and G. J. Williams, Struct. Dyn., 2015, 2, 041701.

5 N. D. Loh and V. Elser, Phys. Rev. E, 2009, 80, 026705.
6 O. Miyashita and Y. Joti, Curr. Opin. Struct. Biol., 2017, 43, 163-169.

7 M. J. Bogan, W. H. Benner, S. Boutet, U. Rohner, M. Frank, A. Barty, M. M. Seibert, F. Maia, S. Marchesini, S. Bajt, B. Woods, V. Riot, S. P. Hau-Riege, M. Svenda, E. Marklund, E. Spiller, J. Hajdu and H. N. Chapman, Nano Lett., 2008, 8, 310-316.

8 M. F. Hantke, D. Hasse, F. R. N. C. Maia, T. Ekeberg, K. John, M. Svenda, N. D. Loh, A. V. Martin, N. Timneanu, D. S. D. Larsson, G. van der Schot, G. H. Carlsson, M. Ingelman, J. Andreasson, D. Westphal, M. Liang, F. Stellato, D. P. DePonte, R. Hartmann, N. Kimmel, R. A. Kirian, M. M. Seibert, K. Mühlig, S. Schorb, K. Ferguson, C. Bostedt, S. Carron, J. D. Bozek, D. Rolles, A. Rudenko, S. Epp, H. N. Chapman, A. Barty, J. Hajdu and I. Andersson, Nat. Photon., 2014, 8, 943-949.

9 Z. Kam, Macromolecules, 1977, 10, 927-934.

10 R. P. Kurta, J. J. Donatelli, C. H. Yoon, P. Berntsen, J. Bielecki, B. J. Daurer, H. DeMirci, P. Fromme, M. F. Hantke, F. R. N. C. Maia, A. Munke, C. Nettelblad, K. Pande, H. K. N. Reddy, J. A. Sellberg, R. G. Sierra, M. Svenda, G. van der Schot, I. A. Vartanyants, G. J. Williams, L. X. Paulraj, A. Aquila, P. H. Zwart and A. P. Mancuso, Phys. Rev. Lett., 2016, 119, 158102.

11 K. Pande, J. J. Donatelli, E. Malmerberg, L. Foucar, B. K. Poon, M. Sutter, S. Botha, S. Basu, R. B. Doak, K. Dörner, S. W. Epp, L. Englert, R. Fromme, E. Hartmann, R. Hartmann, G. Hauser, J. Hattne, A. Hosseinizadeh, S. Kassemeyer, L. Lomb, S. F. C. Montero, A. Menzel, D. Rolles, A. Rudenko, M. M. Seibert, R. G. Sierra, P. Schwander, A. Ourmazd, P. Fromme, N. K. Sauter, M. Bogan, J. Bozek, C. Bostedt, I. Schlichting, C. A. Kerfeld and P. H. Zwart, Sci. Data, 2018, 5, 180201.

12 K. Pande, J. J. Donatelli, E. Malmerberg, L. Foucar, C. Bostedt, I. Schlichting and P. H. Zwart, Proc. Natl. Acad. Sci. USA, 2018, 115, 11772-11777.

13 V. Elser, New J. Phys., 2011, 13, 123014.

14 J. Pérez and Y. Nishino, Curr. Opin. Struct. Biol., 2012, 22, 670678.

15 T. Kimura, Y. Joti, A. Shibuya, C. Song, S. Kim, K. Tono, M. Yabashi, M. Tamakoshi, T. Moriya, T. Oshima, T. Ishikawa, Y. Bessho and Y. Nishino, Nat. Commun., 2014, 5, 3052.

16 R. lida, H. Kawamura, K. Niikura, T. Kimura, S. Sekiguchi, Y. Joti, Y. Bessho, H. Mitomo, Y. Nishino and K. Ijiro, Langmuir, 2015, 31, 4054-4062.

17 J. Wei, K. Niikura, T. Higuchi, T. Kimura, H. Mitomo, H. Jinnai, Y. Joti, Y. Bessho, Y. Nishino, Y. Matsuo and K. Ijiro, J. Am. Chem. Soc., 2016, 138, 3274-3277.

18 C. Song, K. Tono, J. Park, T. Ebisu, S. Kim, H. Shimada, S. Kim, M. Gallagher-Jones, D. Nam, T. Sato, T. Togashi, K. Ogawa, Y. Joti, T. Kameshima, S. Ono, T. Hatsui, S. Iwata, M. Yabashi and T. Ishikawa, J. Appl. Cryst., 2014, 47, 188-197.

$19 \mathrm{H}$. Yumoto et al., in preparation (2019).

20 T. Kimura et al., in preparation (2019).

21 G. M. Whitesides, E. Ostuni, S. Takayama, X. Jiang and D. E. Ingber, Annu. Rev. Biomed. Eng., 2001, 3, 335-373.

22 J. N. Anker, W. P. Hall, O. Lyandres, N. C. Shah, J. Zhao and R. P. Van Duyne, Nat. Mater., 2008, 7, 442-453.

23 T. Ando, T. Uchihashi, S. Scheuring, Chem. Rev., 2014, 114, 3120-3188.

24 A. Ulman, Chem. Rev., 1996, 96, 1533-1554.

25 R. Watanabe, N. Soga, D. Fujita, K. V. Tabata, L. Yamauchi, S. H. Kim, D. Asanuma, M. Kamiya, Y. Urano, H. Suga and H. Noji, Nat. Commun., 2014, 5, 4519.

26 P. A. Midgley and R. E. Dunin-Borkowski, Nat. Mater., 2009, 8, 271-280.

27 Y. Nishino, Y. Takahashi, N. Imamoto, T. Ishikawa and K. Maeshima, Phys. Rev. Lett., 2009, 102, 018101.

28 T. Kameshima, S. Ono, T. Kudo, K. Ozaki, Y. Kirihara, K. Kobayashi, Y. Inubushi, M. Yabashi, T. Horigome, A. Holland, K. Holland, D. Burt, H. Murao and T. Hatsui, Rev. Sci. Instrum., 2014, 85, 033110. 
29 H. Yumoto, H. Mimura, T. Koyama, S. Matsuyama, K. Tono, T. Togashi, Y. Inubushi, T. Sato, T. Tanaka, T. Kimura, H. Yokoyama, J. Kim, Y. Sano, Y. Hachisu, M. Yabashi, H. Ohashi, H. Ohmori, T. Ishikawa and K. Yamauchi, Nat. Photon., 2013, 7, 43-47.

30 Y. Joti, T. Kameshima, M. Yamaga, T. Sugimoto, K. Okada, T. Abe, Y. Furukawa, T. Ohata, R. Tanaka, T. Hatsui and M. Yabashi, J. Synchrotron Rad., 2015, 22, 571-576.

31 J. R. Fienup, Appl. Opt., 1982, 21, 2758-2769.

32 S. Marchesini, H. He, H. N. Chapman, S. P. Hau-Riege, A. Noy, M. R. Howells, U. Weierstall and J. C. H. Spence, Phys. Rev. B, 2003, 68, 140101(R).

33 J. A. Rodriguez, R. Xu, C. C. Chen, Y. Zou and J. Miao, J. Appl. Crystallogr., 2013, 46, 312-318.

34 H. N. Chapman, A. Barty, S. Marchesini, A. Noy, S. P. Hau-Riege, C. Cui, M. R. Howells, R. Rosen, H. He, J. C. H. Spence, U. Weierstall, T. Beetz, C. Jacobsen, and D. Shapiro, J. Opt. Soc. Am. A, 2006, 23, 1179-1200.

35 H. N. Chapman, Annu. Rev. Biochem., 2019, 88, 35-58. 